\title{
Assistentes de Aprendizagem em Simuladores de Voo
}

\author{
Edgar Eler, Orivaldo de Lira Tavares
}

\author{
Laboratório de Informática na Educação \\ Universidade Federal do Espírito Santo (UFES) - Vitória, ES - Brasil \\ elerdedgar.systems, tavareseinf.ufes.br
}

\begin{abstract}
The formation of an individual to the command of an aircraft is based on preparing him to make quick decisions when facing high responsibility situations and subjected to a high-pressure environment due to several factors involved. Given this scenario, it is important that the flight simulation activities prepare the apprentice to the immediate analysis of all the available variables in order to improve the critical decision making. Thereby, it is possible to support the assessment of the apprentice's ability to perform his role as a pilot. This paper discusses the conception of learning assistants for piloting, in order to monitor and interact with the practice of the apprentice in the simulator.
\end{abstract}

Key words: Flight Simulation, Learning Assistant, Multi-Agent System, Aircraft Pilot Formation.

Resumo. A formação de um indivíduo para o comando de uma aeronave tem como base prepará-lo para tomar decisões rápidas diante de situações de elevada responsabilidade e submetido a um ambiente de grande pressão proveniente de diversos fatores envolvidos. Dado esse cenário, é importante que as atividades de simulação de voo preparem o aprendiz para a análise imediata de todas as variáveis disponíveis de forma a agilizar a tomada de decisão crítica. Com isso, é possivel auxiliar a avaliação da aptidão do aprendiz para exercer sua função como piloto. Este trabalho aborda a concepção de assistentes de aprendizagem de pilotagem, de modo a acompanhar e interagir com a prática do aprendiz no simulador.

Palavras-chave: Simulação de Voo, Assistente de Aprendizagem, Sistema Multiagente, Formação de Piloto de Aeronaves.

\section{Introdução}

A formação de indivíduos para exercerem a pilotagem de aeronaves foi aprimorada nas últimas décadas com a utilização dos simuladores, sistemas computacionais que simulam, de forma visual e interativa, a atividade da prática de voo. Por meio da utilização desses simuladores, os primeiros contatos dos aprendizes com o ambiente de pilotagem puderam ser estabelecidos ainda em terra, diretamente no computador, reduzindo o volume de horas de voo necessárias para a formação do piloto, o que impacta na redução dos custos de treinamento e no aumento da segurança.

Em geral, a evolução dos simuladores ao longo dos anos foi focada no realismo operacional e visual, tanto das aeronaves, quanto do ambiente externo. Foram desenvolvidos, inclusive, dispositivos que simulam cabines completas de aeronaves, ampliando a imersão dos aprendizes por meio do alto nível de realismo alcançado na prática da pilotagem de determinada aeronave. 
No entanto, como o foco evolutivo dos simuladores de voo permaneceu na precisão operacional e qualidade visual, a área pedagógica do ensino da pilotagem de aeronaves não fez parte do escopo de desenvolvimento dos simuladores. Isto faz com que as etapas de prática em simulador dos cursos de formação de pilotos profissionais tenham de ser acompanhadas de forma direta e individual pelos tutores, que supervisionam os procedimentos dos aprendizes e analisam seu desenvolvimento prático. $\mathrm{O}$ acompanhamento individual, da prática em ambiente de simulação, limita $\mathrm{o}$ alcance pedagógico dos tutores e, com isso, aumenta o volume de trabalho deles, reduzindo a possibilidade de implementação de estratégias pedagógicas que impactem simultaneamente em todo o grupo de aprendizes.

Buscando uma alternativa computacional para a demanda pedagógica dos simuladores, é apresentado neste trabalho o sistema multiagente denominado Assistente Virtual de Voo VAFlight, que permite aos tutores dos cursos de formação de pilotos de aeronaves a usarem abordagens pedagógicas flexíveis, nas práticas dos aprendizes em simulador de voo. Isso será alcançado com a construção de assistentes de aprendizagem em ambiente web por meio de ferramentas visuais no formato de fluxogramas, que permitam aos tutores a especificação de diretrizes e requisitos de propriedades do voo, para determinado momento da prática em simulador de voo.

\section{Metodologia}

Este trabalho foi desenvolvido por meio de pesquisa experimental que consistiu na criação de sistemas multiagentes a serem utilizados em cursos de formação de pilotos de aeronaves. A metodologia de pesquisa adotada tem as seguintes características: quanto à natureza, é um trabalho científico original; quanto aos objetivos, é uma pesquisa descritiva, um estudo de caso e uma pesquisa experimental; quanto aos procedimentos, é uma pesquisa bibliográfica e experimental; quanto ao objeto, é uma pesquisa bibliográfica, de laboratório e de campo.

Segundo Waslawick (2008), o método é a sequência de passos a serem executados para se atingir determinado objetivo proposto. A Figura 1 ilustra o método utilizado neste trabalho. Na fase de Projeto, são definidos o escopo e os objetivos da pesquisa; na fase de Escrita, são realizadas as pesquisas bibliográficas e os estudos de caso; na fase de Protótipo, são realizadas as pesquisas experimentais e de laboratório que consistem no desenvolvimento e teste dos sistemas multiagentes; na etapa de Homologação, são implementados cenários e situações reais para a constatação dos resultados.

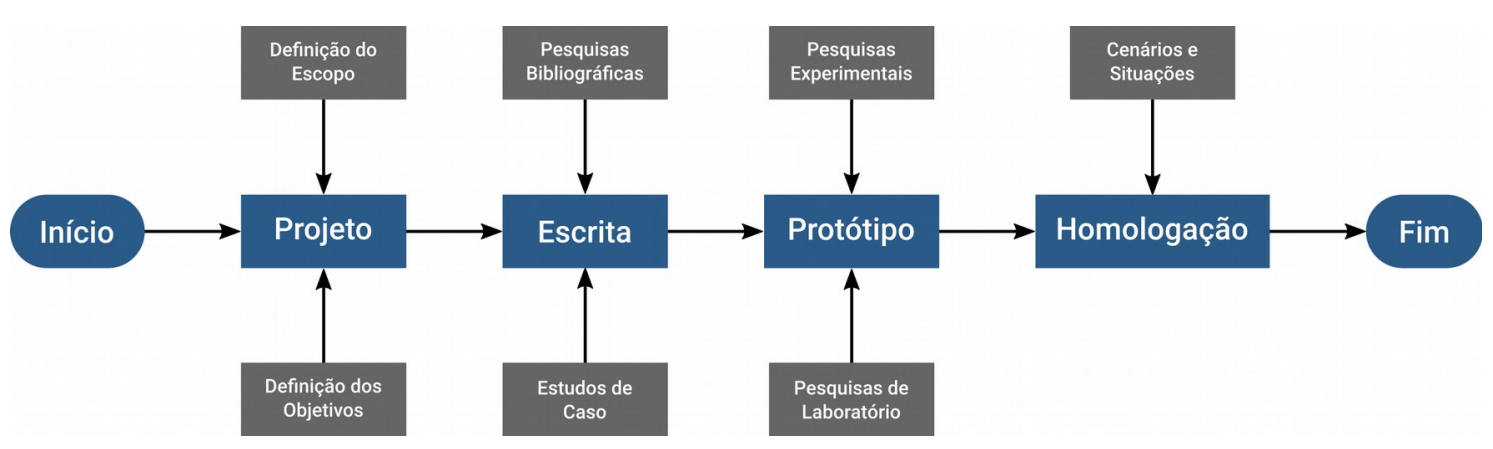

Figura 1. Etapas do Desenvolvimento do Projeto. 
VI Congresso Brasileiro de Informática na Educação (CBIE 2017)

Anais do XXVIII Simpósio Brasileiro de Informática na Educação (SBIE 2017)

\section{Contextualização Literária}

Antes da execução do primeiro voo, acreditava-se que uma aeronave motorizada seria um dispositivo simples de controlar e que exigiria poucas habilidades de controle do piloto. Em pouco tempo, foi constatado que esta suposição estava incorreta, uma vez que as primeiras aeronaves possuíam características únicas e fatais. A necessidade da preparação efetiva de pilotos para as tarefas de voo levaram à ideia de criar dispositivos que poderiam simular as características de uma aeronave, o que seria inestimável como ferramenta de treinamento. Esta foi a concepção da simulação de voo. (Lawn, 1998)

\subsection{A simulação de voo}

Segundo Gheorghiu (2013), a simulação é um método usado na aviação composto por um conjunto de tecnologias que reproduzem a interação homem-aeronave para propósitos de treinamento, avaliação de desempenho, pesquisa e desenvolvimento. Se refere, primeiramente, à criação de representações físicas do terreno para reproduzir elementos técnicos e os comportamentos de uma aeronave sob várias condições e em interação com o elemento humano.

Ruiz, Aguado e Moreno (2014) listam alguns dos principais recursos que quaisquer tipos de simulações possuem: são úteis para tomada de decisões; permitem a avaliação e a previsão de potenciais soluções futuras; permitem a otimização do comportamento do sistema e oferecem um método seguro e econômico de treinamento de operadores. Informam, ainda, que o componente de simulação em uma experiência de aprendizagem permite levar pessoas ao tempo ou lugar que seria impossível ou provavelmente inviável de praticar diretamente, incentivando assim a aprendizagem dentro de situações artificiais que podem ser totalmente adaptadas para aprimorar a experiência de aprendizagem. Adicionalmente, os recursos já listados podem ser usualmente complementados com a capacidade dos simuladores de representar e conectar grandes volumes de informações por meio de multimídia, como imagens em alta definição e som ambiente altamente fiel, o que torna a experiência de aprendizagem mais atrativa aos estudantes.

Entretanto, segundo Nählinder, Berggren e Persson (2005), a solução de treinamento mais ideal pode se distanciar um pouco da necessidade por simular o mundo tão realístico quanto possível. Acredita-se que simuladores podem aumentar a eficiência do treinamento dramaticamente quando incorporam abordagens pedagógicas como complemento à fidelidade. Ao fornecer uma série de recursos pedagógicos que estão de acordo com a cultura de treinamento, um simulador de treinamento pode provavelmente ser muito mais eficiente do que um simulador com fidelidade quase perfeita.

A partir desta premissa, podem ser propostas soluções como os sistemas associados autônomos, descritos por Levi et al. (1992), que auxiliam um piloto humano a realizar uma missão, por meio da obtenção e compilação de informações, recomendação de ações e, em alguns casos, desempenhando determinadas tarefas da missão.

\subsection{A abordagem pedagógica}

Freitas e Neumann (2009) informam que, por causa do imediatismo e do apelo amplo de aprendizagem em mundos imersivos, o uso de modelagem e simulações computacionais para treinamento estão induzindo uma reconsideração de como os indivíduos aprendem, 
onde aprendem, o que aprendem e quando aprendem. Isto está levando à revisão das abordagens de aprendizagem, tanto para contextos formais e informais, quanto para contextos mistos. Porém, apesar de muitos aprendizes estarem usando estas ferramentas de forma diária, tem havido muito pouca consideração de mudanças nos modelos pedagógicos que sustentam essas novas formas de organizar a aprendizagem.

Como possíveis estruturas que abranjam essas mudanças em modelos pedagógicos, Carvalho, Nevado e Menezes (2005) propõem as arquiteturas pedagógicas, que são estruturas de aprendizagem compostas pela abordagem pedagógica, software, internet, inteligência artificial, educação à distância e concepção de tempo e espaço. Acolhem didáticas flexíveis, maleáveis e adaptáveis a diferentes temas. As perspectivas de tempo e espaço de aprendizagem passam a se moldar aos ritmos impostos pelo sujeito que aprende, removendo a exigência pela localização em escola ou sala de aula. As arquiteturas funcionam como mapas, mostrando diferentes possibilidades de se realizar algo, fornecendo ao aprendiz a escolha dos caminhos a serem percorridos. Os professores são essenciais para a criação e reinvenção dessas arquiteturas pedagógicas. Já os aprendizes atuam como protagonistas, agindo e refletindo sobre experiências com a orientação do professor.

\section{Ambientes Computacionais para Assistência de Aprendizagem em Simuladores}

Nos simuladores de voo, a assistência de aprendizagem é composta principalmente por missões ou tutoriais pré-instalados pelos fabricantes. Os assistentes são procedimentos baseados em passo a passo, de modo a orientar o aprendiz a concluir um passo para que o próximo seja apresentado. Além disso, existem projetos que abordam outros ambientes de simulação (diferente da prática de voo), como os ambientes descritos por Dzikovska et al. (2014) e Zachary et al. (1999), mas que possuem abordagens pedagógicas com foco na melhoria do desenvolvimento dos aprendizes e podem ser considerados na análise dos ambientes computacionais para assistência de aprendizagem em simuladores. A seguir, são descritos estes ambientes computacionais.

\subsection{Assistentes de Aprendizagem do Microsoft Flight Simulator X - Missões}

O Microsoft Flight Simulator X (FSX) é um dos simuladores de voo comerciais mais tradicionais do mercado. Segundo Lackey (2006), o atrativo principal desta versão do simulador é um módulo de criação de missões poderoso e simples de usar, utilizado para elaborar as 50 missões que vêm embarcadas no jogo. As missões embarcadas vão desde as missões relativamente simples, como procurar por um campista perdido em montanhas nevadas, até as mais desafiadoras, em tarefas frenéticas como resgatar trabalhadores de uma plataforma de petróleo explodindo. Segundo Stark (2008), esse módulo fornece uma oportunidade de ensinar habilidades e explorar os recursos e efeitos do FSX, de uma maneira desafiadora e divertida.

\subsection{Assistentes de Aprendizagem do FlightGear - Tutoriais}

FlightGear é um simulador de voo livre, desenvolvido cooperativamente via internet, por um grupo de entusiastas de simulação de voo e de programação. O FlightGear contém um sistema tutorial sobre voo, onde um instrutor simulado fornece lições virtuais. Essas lições variam entre aeronaves, desde simples tutoriais ensinando como iniciar os motores em uma aeronave, até lições completas ensinando como voar pela primeira vez. Cada tutorial consiste em um número de passos discretos que o aprendiz 
VI Congresso Brasileiro de Informática na Educação (CBIE 2017)

Anais do XXVIII Simpósio Brasileiro de Informática na Educação (SBIE 2017)

precisa completar. O instrutor simulado fornece as orientações de como completar cada passo, e observa como o aprendiz o realizou, fornecendo instruções adicionais caso necessário. (BASLER et al., 2015)

\subsection{BEETLE II - Entendimento de Linguagem Natural e Geração Automática de Respostas}

Segundo Dzikovska et al. (2014), o Ambiente de Aprendizagem Tutorial de Eletricidade e Eletrônica Básica (BEETLE II) é um Sistema Tutorial Inteligente (ITS), focado no desenvolvimento de um currículo efetivo para os subtópicos de física, eletricidade e circuitos elétricos, no domínio da Ciência, Tecnologia, Engenharia e Matemática (STEM). As lições no BEETLE II aplicam uma abordagem instrucional, implementada com base na análise do comportamento de um tutor humano para o mesmo currículo, dentro do contexto de uma bancada de circuito simulada. Para cada tópico, os estudantes são questionados a prever o comportamento de dado circuito e explicar sua previsão. Em seguida, os estudantes testam suas previsões no simulador de circuito construindo os circuitos e observando seus comportamentos. Depois que o teste é completado, os estudantes são questionados se os resultados coincidiram com suas previsões. Finalmente, quando as previsões não se concretizam, o sistema solicita aos estudantes que tentem e gerem uma explicação para o comportamento do circuito que eles acabaram de observar. Esse fluxo geral é denominado estratégia de "PrevisãoVerificação-Avaliação" (PVE). A maior parte do currículo é construída dessa forma, o que encoraja cada estudante a pensar profundamente sobre os fenômenos que ele observa e tentar inferir os princípios explicativos fundamentais do domínio.

\subsection{AETS - Sistema de Treinamento Embarcado Avançado}

O Sistema de Treinamento Embarcado Avançado (AETS) é um projeto de Demonstração de Tecnologia Avançada, conduzido pela Marinha dos Estados Unidos, para aplicar tecnologias de tutoria inteligente no treinamento de tripulação dos seus navios. O AETS buscou aplicar os conceitos de tutoria inteligente - aprendizagem baseada em problemas, diagnósticos cognitivos, modelagem de estudante, e tutoria adaptativa e focada - mas encontrou novas abordagens a serem desenvolvidas para lidar com a indeterminação, espaços de conhecimento abertos, solução de problemas de equipe e a natureza de tempo real dos domínios táticos. Entre os princípios de projeto do AETS, estão: exigir menos instrutores; apoiar treinamentos mais consistentes; permitir um melhor rastreamento e análise e fornecer instruções aprimoradas e retornos sobre a performance da equipe. Ao invés de identificar porque os aprendizes cometem erros, o AETS irá tentar determinar como fazer para que eles aprendam o caminho correto. (ZACHARY et al., 1999)

\section{VAFlight - Assistente Virtual de Voo}

No início dos cursos de formação de indivíduos para pilotagem de aeronaves, é requisito que o aprendiz construa um conhecimento teórico a respeito das características do voo, regulamentos de tráfego aéreo, meteorologia aeronáutica, conhecimentos técnicos $\mathrm{e}$ motores, além de legislações aeronáuticas, práticas de segurança de voo, regulamentação, medicina e direito aeroespacial. A partir do momento em que o aprendiz conclui sua formação teórica inicial, ele já tem base de conhecimento suficiente para iniciar seu processo de aprendizagem por meio da prática na operação de aeronaves em simuladores de voo. 
Uma vez que o aprendiz esteja na etapa de prática em simulador de voo, cabe ao tutor humano a tarefa de supervisionar essa prática. Esse processo de supervisão consome tempo e limita a atuação e abordagem pedagógica do tutor. Dadas as particularidades na construção do conhecimento prático de cada aprendiz, a supervisão do tutor humano tem de ser individual. A possibilidade da configuração de assistentes tradicionais no modelo procedimental, como descrito no capítulo 3 , auxilia o tutor na aplicação de exercícios práticos em determinado grupo de aprendizes. Porém, esses assistentes limitam o protagonismo dos aprendizes na formação de sua aprendizagem, pois toda a evolução de determinado exercício prático já foi pré-determinado pelo tutor, exigindo-se que cada passo seja seguido exatamente como previsto para que o próximo passo seja alcançado.

Para a criação de um ambiente que favoreça o protagonismo do aprendiz na formação de sua aprendizagem prática em simulador de voo, é considerada no sistema multiagente deste trabalho, denominado Assistente Virtual de Voo VAFlight, uma arquitetura pedagógica com abordagem flexível, na qual o aprendiz tenha liberdade na realização de sua prática, sendo supervisionado pelo assistente de aprendizagem configurado pelo tutor. Existem outras abordagens pedagógicas possíveis, porém, dada a característica procedimental intrínseca no domínio do conhecimento da operação de aeronaves, entende-se como válido, além de aderente à expectativa atual dos tutores, o uso dos assistentes de aprendizagem enquanto supervisores da prática do aprendiz no simulador de voo.

\subsection{Os Assistentes de Aprendizagem}

Os assistentes de aprendizagem do sistema VAFlight são configurados pelos tutores por meio de uma linguagem de programação visual, criando procedimentos compostos por ações e condições. Em cada ação ou condição, podem ser consideradas ou validadas as propriedades de voo em determinado momento da prática do aprendiz. Com base nessas propriedades e nos passos configurados no assistente, o sistema analisa a prática do aprendiz e verifica se ele cumpriu ou não os critérios de determinada condição. A arquitetura pedagógica possibilita ao tutor que aplique uma abordagem pedagógica flexível, na qual, caso o aprendiz não cumpra determinado passo, o assistente não imponha a ele a necessidade pelo cumprimento, mas sim questione a razão que o levou a tal atitude, permitindo ao aprendiz a escolha do caminho de sua aprendizagem.

Os procedimentos dos assistentes de aprendizagem são configurados visualmente por meio de fluxogramas, nos quais os tutores podem criar as ações e condições e vinculá-las no diagrama, conforme exemplo ilustrado na Figura 2, sendo as ações representadas pelos retângulos, as condições representadas pelos losangos, o início e fim do assistente representados pelas elipses e os vínculos representados pelas setas. Nessa ilustração, após o início de sua execução, o assistente verifica se o motor da aeronave está ligado. Caso o aprendiz não ligue o motor dentro de 5 minutos, o assistente questiona o aprendiz sobre o motivo. Caso a resposta do aprendiz seja coerente, como, por exemplo, se ele ainda estiver realizando uma checagem pré-voo, o assistente volta ao início da contagem e verificação do motor ligado. Caso a resposta recebida não proceda, o assistente questiona novamente ao aprendiz sobre o motivo do motor ainda não estar ligado, até que a resposta seja coerente, ou até que o aprendiz ligue o motor. Caso o aprendiz ligue o motor, o assistente de aprendizagem de decolagem passa a analisar se a aeronave já atingiu a altitude de cruzeiro. Enquanto não atingir, são verificadas outras propriedades de voo, com os devidos questionamentos específicos. Caso atinja, o assistente de decolagem é finalizado. 


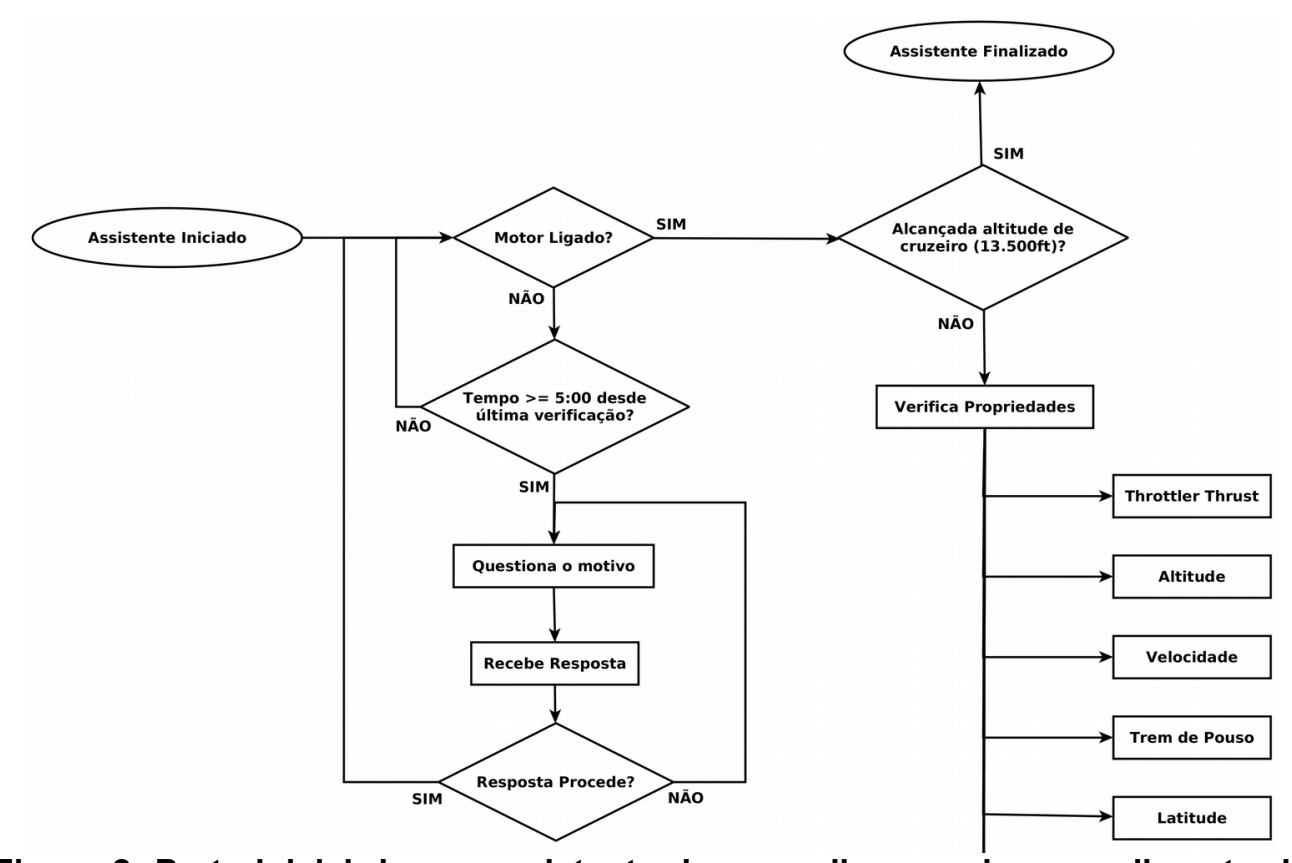

Figura 2. Parte inicial de um assistente de aprendizagem do procedimento de decolagem.

O tutor tem autonomia para configurar quantos assistentes forem necessários para determinada prática no simulador. $\mathrm{O}$ aprendiz pode, por exemplo, estar em uma prática de voo livre no simulador e o tutor configurar um assistente de aprendizagem que, em determinado momento, altere as propriedades de voo, de modo que surja fogo em um dos motores da aeronave. Nesse momento, o aprendiz tem que lidar com a situação emergencial e o assistente pode auxiliá-lo, questionando quais são os passos que o aprendiz irá realizar e qual a razão para a escolha desses passos. O tutor pode configurar um procedimento padrão para solução emergencial desse problema, e compará-lo com as ações escolhidas pelo aprendiz. Como forma de garantir o protagonismo do aprendiz na formação de sua aprendizagem, o assistente não intervém nas ações do aprendiz, nem mesmo demonstra o caminho a ser seguido por ele, mas sim observa as ações do mesmo e questiona seus motivos, armazenando suas respostas. Caso o tutor considere as ações adotadas pelo aprendiz mais eficientes do que as configuradas por ele, é possível implementá-las como melhorias para o assistente de aprendizagem. Essa eficiência pode ser medida com respeito a alguns critérios, como tempo de manobra, consumo de combustível, entre outros.

\section{Estrutura Computacional}

Para o uso dos assistentes de aprendizagem, em um grupo de estudantes de cursos de formação de pilotos de aeronaves, o tutor precisa de um ambiente que centralize a configuração dos assistentes e das simulações a serem realizadas, além de integrar múltiplas estações de simulação, de forma simultânea ou não. Adicionalmente, caso o tutor deseje acolher uma didática flexível, adaptando-se ao espaço e tempo do aprendiz, conforme a proposta deste projeto, é necessária uma estrutura distribuída via internet.

A integração dos assistentes de aprendizagem do sistema VAFlight é suportada por uma arquitetura distribuída, composta por agentes de software executando em um servidor central e nas estações de simulação. A integração desses agentes de software é feita via internet. A Figura 3 ilustra a arquitetura dos agentes de software integrados via internet. Essa infraestrutura do sistema VAFlight é descrita a seguir. 
VI Congresso Brasileiro de Informática na Educação (CBIE 2017)

Anais do XXVIII Simpósio Brasileiro de Informática na Educação (SBIE 2017)

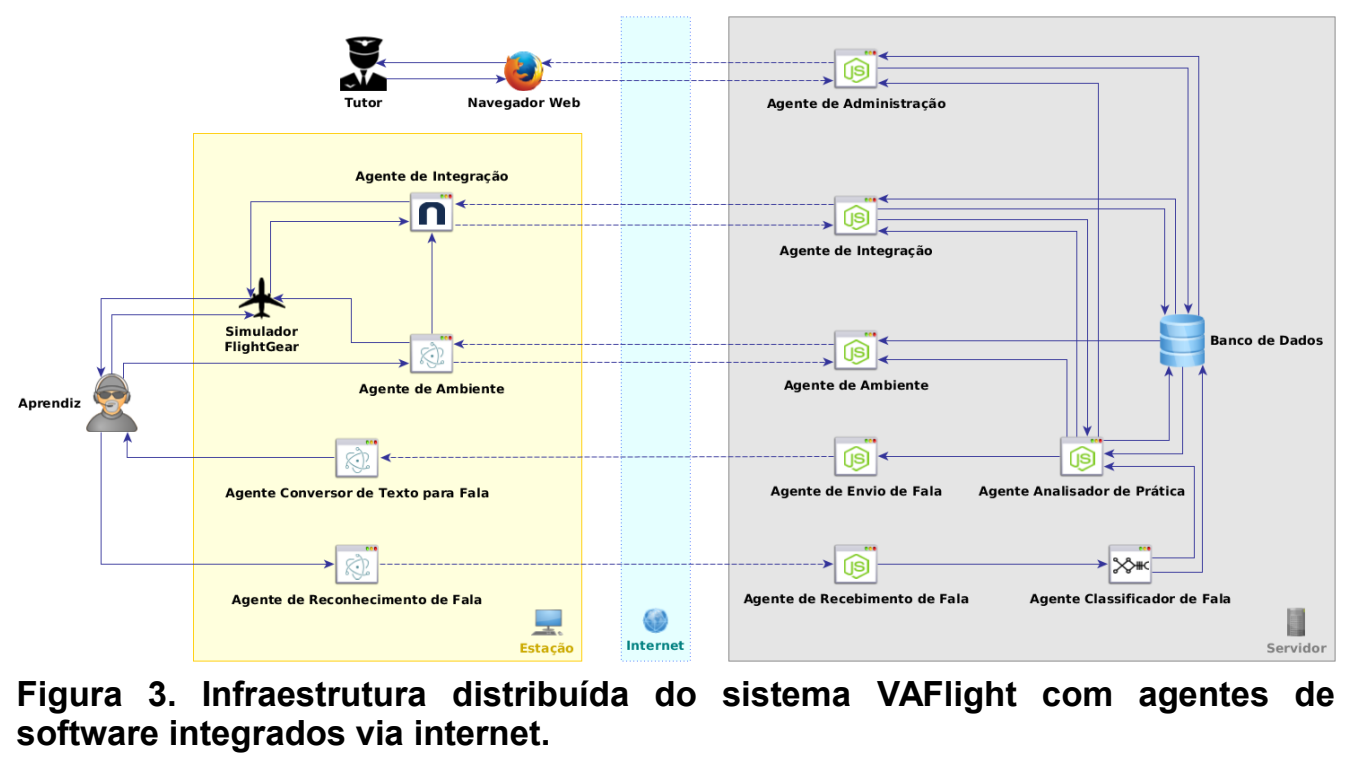

O tutor acessa o agente de administração, localizado no servidor, por meio do navegador web. Com o agente de administração o tutor pode criar novos assistentes de aprendizagem e ambientes de simulação, como, por exemplo, a aeronave a ser utilizada, em qual aeródromo é iniciada a simulação, data e hora, quais as condições meteorológicas no momento, entre outros. $\mathrm{O}$ agente de administração armazena esses novos assistentes de aprendizagem e ambientes de simulação no banco de dados.

O aprendiz acessa o agente de ambiente na estação para iniciar sua simulação. $\mathrm{O}$ agente de ambiente na estação acessa o agente de ambiente no servidor para capturar os dados de configuração da simulação a ser executada. Ao capturar os dados da simulação, o agente de ambiente na estação executa o simulador FlightGear parametrizado com a simulação específica para o aprendiz. A partir deste momento, o aprendiz inicia a prática de simulação e interage com o FlightGear. O simulador FlightGear foi escolhido por possuir código-fonte aberto, ser amplamente documentado e possuir uma linguagem de integração robusta.

Uma vez que o aprendiz inicie sua prática no FlightGear, o agente de integração na estação inicia o processo de captura das propriedades de voo e envio para o agente de integração no servidor. $\mathrm{O}$ agente de integração no servidor recebe as propriedades, as armazena no banco de dados e as envia para o agente analisador de prática. A Figura 4 ilustra a sincronização dos agentes de integração.

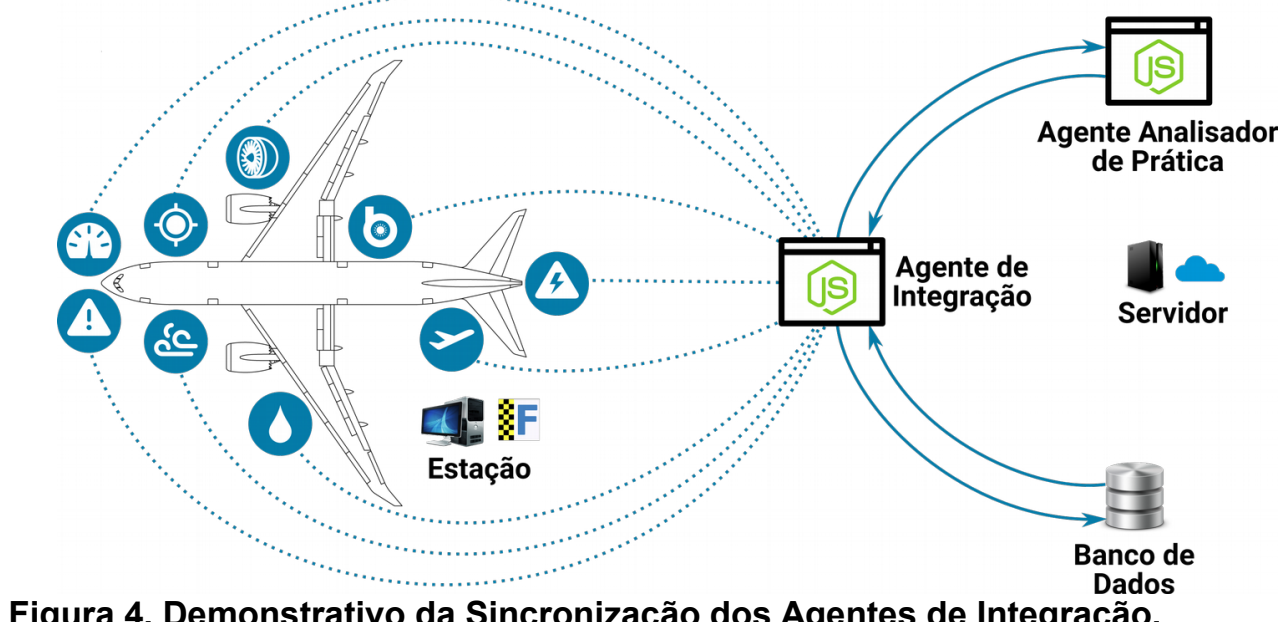


O agente analisador de prática recebe as propriedades do agente de integração e as analisa junto aos passos configurados pelo tutor nos assistentes de aprendizagem. Assim que necessário, o agente analisador de prática envia interações em fala, por meio do agente de envio de fala, além de interações no simulador, por meio do Agente de Integração.

O agente conversor de texto para fala da estação captura os textos enviados pelo agente de envio de fala do servidor e os converte para áudio, conversando diretamente com o aprendiz. Caso o aprendiz responda via fala a interação recebida, o agente de reconhecimento de fala da estação transforma essa fala em texto e o envia para o agente de recebimento de fala do servidor. $\mathrm{O}$ agente de recebimento de fala encaminha os textos para o agente classificador de fala, que identifica os padrões, armazena a interação do aprendiz no banco de dados e a envia para o agente analisador de prática. Caso o aprendiz responda via comandos no simulador FlightGear, os agentes de integração na estação e no servidor armazenam essas ações.

O agente analisador de prática realiza a análise do padrão da resposta recebida do aprendiz, seja via fala ou comandos no simulador, e verifica se a ação realizada está compatível com o procedimento configurado no assistente de aprendizagem. Caso a ação do aprendiz resulte em falha catastrófica, o agente analisador de prática envia um comando ao agente de ambiente do servidor para que reinicie a simulação no agente de ambiente da estação.

Todo o ambiente do sistema VAFlight foi implementado e os testes iniciais foram realizados, confeccionando-se assistentes de aprendizagem de decolagem, de pouso e de falha em motor durante o voo. Com isso, a proposta do sistema foi validada. Os testes continuarão sendo realizados para encontrar pontos de ajuste e de aperfeiçoamento do ambiente implementado.

\section{Perspectivas Futuras e Considerações Finais}

O sistema VAFlight pode viabilizar a existência de disciplinas à distância, para as simulações de voo dos cursos de formação de pilotos de aeronaves, uma vez que os tutores não precisam acompanhar fisicamente e individualmente os aprendizes em sua prática. Pelo fato dos assistentes de aprendizagem serem previamente configurados, torna-se viável a adoção da modalidade não presencial durante as simulações. A viabilização dessa etapa em ambiente não presencial, aliada às etapas teóricas dos cursos de formação nessa mesma modalidade, facilita a formação de novos pilotos de aeronaves, pois reduz a necessidade da presença física dos tutores e permite que os estudantes escolham tanto o local quanto o horário para a realização dos estudos, uma vez que podem utilizar seus próprios computadores para as etapas iniciais de simulação.

Para que o sistema VAFlight seja amplamente usado e validado, ele será oferecido, sem custos, para instituições de ensino civis e militares que ofertem cursos de formação de pilotos de aeronaves. Essa oferta é possível uma vez que o simulador de voo FlightGear é gratuito e não possui restrições de uso em sua licença.

A adequação da abordagem pedagógica das etapas de prática em simulador dos cursos de formação de pilotos de aeronaves, com os avanços tecnológicos atuais, pode fornecer, tanto aos tutores quanto aos aprendizes, uma experiência de aprendizagem mais consistente e flexibilizar as estruturas didáticas vigentes atualmente.

A adição de novos agentes de software, tanto de interação com o aprendiz quanto de análise de sua prática, pode aprimorar ainda mais a experiência de voo 
VI Congresso Brasileiro de Informática na Educação (CBIE 2017)

Anais do XXVIII Simpósio Brasileiro de Informática na Educação (SBIE 2017)

simulado do aprendiz, aproximando-o do que seria um ambiente físico de laboratório de simulação de voo supervisionado por um tutor humano.

A adoção do sistema VAFlight pode, num futuro próximo, aliado com as tecnologias de ensino a distância já estabelecidas, possibilitar a existência de cursos de formação de pilotos de aeronaves cuja presença física dos aprendizes só seja exigida na última etapa do curso: na prática de voo realizada diretamente na aeronave.

\section{Referências}

Basler, M. et al. (2015) "The FlightGear Manual - For FlightGear version 3.4.0", http://mapserver.flightgear.org/getstart, Março.

Carvalho, M.J.S., Nevado, R.A. e Menezes, C.S. (2005). “Arquiteturas pedagógicas para educação à distância: concepções e suporte telemático", Anais - XVI Simpósio Brasileiro de Informática na Educação, 1, 362-372.

Dzikovska, M. et al. (2014). "BEETLE II: Deep Natural Language Understanding and Automatic Feedback Generation for Intelligent Tutoring in Basic Electricity and Electronics", In: International Journal of Artificial Intelligence in Education, vol. 24, p. 284-332.

Freitas, S. e Neumann, T. (2009) "The use of 'exploratory learning' for supporting immersive learning in virtual environments", In: Computers \& Education, issue 52, p. 343-352.

Gheorghiu, A. (2013) "Flight Simulation in Air Force Training. A Knowledge Transfer Efficiency Perspective", In: Journal of Defense Resources Management, vol. 4, p. 153-158, Transylvania University of Brasov.

Lackey, J. (2006) "Microsoft Flight Simulator - Flying the friendlier skies", In: Computer Gaming World, issue 267, p. 64-64.

Lawn, P. (1998) "The Enhancement of a Flight Simulator System with Teaching and Research Applications", Dissertação de Mestrado, The Department of Mechanical Engineering, Concordia University, Montreal, Quebec, Canada.

Levi, K.R. et al. (1992) "An Explanation-Based-Learning Approach to Knowledge Compilation - A Pilot's Associate Application", In: IEEE Expert, vol. 7, issue 3, p. 44-51.

Nählinder, S., Berggren, P. e Persson, B. (2005) "Increasing Training Efficiency Using Embedded Pedagogical Tools in a Combat Flight Simulator", In: Proceedings of the Human Factors and Ergonomics Society 49th Annual Meeting, p. 2197-2200.

Ruiz, S., Aguado, C. e Moreno, R. (2014) "Educational Simulation in Practice: A Teaching Experience Using a Flight Simulator", In: Journal of Technology and Science Education, vol. 3, p. 181-200, Barcelona, Espanha.

Stark, P. (2007) "FSX Mission Building - Tools of the Trade", In: PC Pilot, issue 48, p. 40-43.

Waslawick, R.S. (2008), Metodologia de Pesquisa para Ciência da Computação, Elsevier, $1^{\mathrm{a}}$ edição.

Zachary, W. et al. (1999) "The Advanced Embedded Training System (AETS): An Intelligent Embedded Tutoring System for Tactical Team Training", In: International Journal of Artificial Intelligence in Education, vol. 10, p. 257-277. 